to me that the theorem in regard to the enneadic centre subsists for a system of 9 points such as referred to in the statement; but that if by possibility the statement be too general, the theorem must, at all erents, subsist for a more special system of 9 points; and that there certainly exist systems of 10 points, such that each 9 of the points have as an enneadic centre the tenth point. [ $I$ have since ascertained that if a quartic surface with 10 nodes has a single node $(3,3)$, the surface is a symmetroid; whence, by what precedes, the remaining nine nodes are each of them $(3,3)$. Added 25th March.]

124. I notice, as a subject of investigation, the following system of correspondence; viz., given any 8 points in space: then to every point in space corresponds a line through this point: viz., the nintb line of the ennead obtained by joining the point with the 8 given points respectively, and to each line in space a point or points on the line : viz., the point or points for each of which the line is the ninth line of the ennead obtained by joining the point with the eight given points respectively.

Dr. Hirst next entered into an explanation of his paper "On the Polar Correlation of two Planes, and its connection with their Quadric Correspondence."

Profs. Cayley, Smith, Mr. Cotterill, and Dr. Hirst took part in the discussions on the papers.

The following presents were made to the Library :-

"On the determination of the Orbit of a Planet from three Observations." By A. Cayley, F.R.S.: from the author.

"Géométrie Supérieure." By M. Chasles : from the President.

Jan. 12th, 1871.

W. SPOTTISWOODE, Esq., F.R.S., President, in the Chair.

Mr. R. B. Hayward, M.A., late Fellow of St. John's College, and Mathematical Master at Harruw, and the Rev. J. Wolstenholme, M.A., Fellow of Christ's College, were proposed for election.

Mr. Walker gave an account of the following paper:-

On Systems of Tangents to Plane Cubic and Quartic Curves.

1. By a "System of Tangents" I understand all the tangents which can be drawn from a point $\left(x_{1} y_{1} z_{1}\right)$ to a proper plane carre (U). According to Dr. Salmon's notation, the equation to the system of tangents drawn to a cubic curve is obtained by equating with zero the discriminant of the binary cubic in $\lambda, \mu$, (Higher Plane Curves, Ed. 1, p. 68,)

$$
\mathrm{U} \lambda^{3}+\Delta_{1} \lambda^{2} \mu+\Delta \lambda \mu^{3}+U_{4} \mu^{3},
$$


where $\Delta_{1}$ stands for $x_{1} \frac{d U}{d x}+y_{1} \frac{d U}{d y}+z_{1} \frac{d U}{d z}$, and $\Delta$ for

$$
x_{1}^{2} \frac{d^{2} \mathrm{U}}{d x^{2}}+y_{1}^{2} \frac{d^{2} \mathrm{U}}{d y^{2}}+z_{1}^{2} \frac{d^{2} \mathrm{U}}{d z^{2}}+2 y_{1} z_{1} \frac{d^{2} \mathrm{U}}{d y d z}+2 z_{1} x_{1} \frac{d^{2} \mathrm{U}}{d z d x}+2 x_{1} y_{1} \frac{d^{2} \mathrm{U}}{d x d y} ;
$$

while $U_{1}$ is the result of substituting $x_{1}, y_{1}, z_{1}$ for $x, y, z$ respectively in U. It will be convenient, in what follows, to use some symbol to represent the operatur $x_{1} \frac{d}{d x}+\ldots$ apart from its subject. Supposing then

$$
\mathrm{D}_{1}=x_{1} \frac{d}{d x}+y_{1} \frac{d}{d y}+z_{1} \frac{d}{d z}
$$

the cubic above in $\lambda, \mu$ would be

$$
U \lambda^{3}+D_{1} U \lambda^{2} \mu+\frac{1}{2} D_{1}^{2} U \lambda \mu^{2}+U_{1} \mu^{3} \text {. }
$$

2. As all the curves, wbich I shall have occasion to notice in this short paper, will be expressed in terms of $U, D_{1} U, \ldots U_{1}$, I have, at the kind suggestion of Prof. Cayley, substituted for these cumbrous symbols the single letters $a, b, c, d$ with numerical coefficients; viz., in the case of cubic curves,

$$
\begin{aligned}
\mathrm{U} & =a, \\
\mathrm{D}_{1} \mathrm{U} & =3 b, \\
\frac{1}{2} \mathrm{D}_{1}^{2} \mathrm{U} & =3 c, \\
\mathrm{U}_{1} & =d ;
\end{aligned}
$$

so that $a$ is the given cubic function of $x y z, b$ a quadric, $c$ a linear function, and $d$ a constant. The cubic in $\lambda, \mu$ will now take the familiar form $a \lambda^{3}+3 b \lambda^{2} \mu+3 c \lambda \mu^{2}+d \mu^{3}$, the discriminant of which is equal to

$$
(a d-b c)^{2}-4\left(a c-b^{2}\right)\left(b d-c^{2}\right)
$$

or, after multiplication by $d^{2}$,

$$
\left(a d^{2}-3 b c d+2 c^{3}\right)^{2}+4\left(b d-c^{2}\right)^{3}
$$

The geometrical interpretation of this discriminant has, as far as $I$ am aware, only been noticed hitherto when it is arranged in the form

$$
\left(a d^{3}-6 b c d+4 c^{3}\right) a+\left(4 b d-3 c^{2}\right) b^{2}
$$

3. From (1) it follows that the equation to the six tangents from $(x, y, z)$ to the cubic $U$ or $a$, may be thrown into the form

$$
u_{2}^{2}-4 u_{4} u_{2}=0
$$

the suffixes indicating the order of the functions in $x y z$; and where

$$
\begin{aligned}
& u_{4}=a c-b^{2} \\
& u_{3}=a d-b c \\
& u_{2}=b d-c^{2}
\end{aligned}
$$

Operating with

$$
\begin{gathered}
x_{1} \frac{d}{d x}+g_{1} \frac{d}{d y}+z_{1} \frac{d}{d z}, \text { or } \mathrm{D}_{1}, \\
\mathrm{D}_{1} a=\mathrm{D}_{1} \mathrm{U}=3 b, \\
\mathrm{D}_{1} b=\frac{1}{3} \mathrm{D}_{1}^{2} \mathrm{U}=2 c, \\
\mathrm{D}_{1} c=\frac{1}{6} \mathrm{D}_{1}^{3} \mathrm{U}=d=\mathrm{U}_{1} ;
\end{gathered}
$$


consequently $\mathrm{D}_{1} u_{4}=a \mathrm{D}_{1} c+c \mathrm{D}_{1} a-2 b \mathrm{D}_{1} b$

$$
=a d+3 b c-4 b c=a d-b c=u_{3} \text {. }
$$

Again $\quad \mathrm{D}_{1} u_{3}=d \mathrm{D}_{1} a-b \mathrm{D}_{1} c-c \mathrm{D}_{1} b$

$$
=3 b d-b d-2 c^{2}=2\left(b d-c^{2}\right)=2 u_{2} \text {. }
$$

Lastly $\quad \mathrm{D}_{1} u_{2}=d \mathrm{D}_{1} b-2 c \mathrm{D}_{1} c=2 c d-2 c d=0$.

If $a, b, c,\left(D_{1} \mathrm{U}\right), \ldots$ represent the results of substituting $x_{1}, y_{1}, z_{1}$ for $x, y, z$ respectively in $a \ldots D_{1} \mathrm{U} \ldots$, then, as is well known,

$$
\begin{aligned}
& a_{1}=\mathrm{U}_{1}=d, \\
& b_{1}=\frac{1}{3}\left(\mathrm{D}_{1} \mathrm{U}\right)_{1}=\mathrm{U}_{1}=d, \\
& c_{1}=\frac{1}{6}\left(\mathrm{D}_{1}^{2} \mathrm{U}\right)_{1}=\mathrm{U}_{1}=d,
\end{aligned}
$$

from which it at once appears that each of the three curves $u_{4}=0$, $u_{3}=0, u_{2}=0$ passes through the point $x_{1} y_{1} z_{1}$ from which the tangents are drawn.

Since $\left(x_{1} y_{1} z_{1}\right)$ is a point on $u_{2}$, and $D_{1} u_{2}$ vanishes identically, $u_{2}$ represents a pair of right lines drawn through that point; and since $\mathrm{D}_{1} u_{4}=u_{3}, \mathrm{D}_{1} u_{3}=(2) u_{2},\left(x_{1} y_{1} z_{1}\right)$ is a double point on $u_{4}$ and its first polar $u_{3}$, while $u_{2}$ is the pair of tangents to each of those curves at the double point.

4. The curves $u_{6}, u_{8}$ having a common double point and common tangents at that point, will meet again in six points, and the form of (4) shows that at these six points the six tungents to $U$ drawn from $\left(x_{1} y_{1} z_{1}\right)$ touch the quartic $u_{4}$.

Further, the form of $u_{4}(5)$ shows that it touches $U$ in the six points in which the latter is met by its first polar $b$; and, therefore, that $u_{4}, \mathrm{U}$ have the same six points of contact with their common tangents. Otherwise it appears from (6) that the nine points of intersection of $U$ and $u_{B}$ lie on the conic $b$, and the line $c$.

The conic $b$ meets $u_{4}$ in two other points besides those referred to above. Equation (5) shows that the line $c$ is a double tangent to $u_{4}$ at those points ; while (7) shows that at the same two points $u_{4}$ is met again by the two tangents at its double point $\left(x_{1} y_{1} z_{1}\right)$, which are also tangents to the conic $b$.

When the point $\left(x_{1} y_{1} z_{1}\right)$ is on $U$, this becomes a point in which two branches of $u_{4}$ touch, $c$ being the tangent at that point both to $U$ and $u_{4}$, which touch in four other points lying on the conic $b$.

(5.) The form (2) of the discriminant gives the equation to the six tangents from $\left(x_{1} y_{1} z_{1}\right)$ to $U$ in the shape

$$
\begin{array}{ll} 
& v_{3}^{2}+4 u_{2}^{3}=0, \\
\text { where } & v_{2}=a d^{2}-3 b c d+2 c^{3}, \\
\text { and, as before, } & u_{2}=b d-c^{2} ;
\end{array}
$$

provided the point $x_{1} y_{1} z_{1}$ be not taken on the curve $U$.

Of course $\left(x_{1} y_{1} z_{1}\right)$ is a point on $v_{3}$. Moreover,

$$
\mathrm{D}_{1} v_{3}=d^{2} \mathrm{D}_{1} a-3 b d \mathrm{D}_{1} c-3 c d \mathrm{D} b+b c^{2} \mathrm{D}_{1} c
$$


but $\mathrm{D}_{1} a=3 b, \mathrm{D}_{1} b=2 c, \mathrm{D}_{1} c=d$, whence

$$
\mathrm{D}_{1} v_{8} \equiv 0 \text {, }
$$

so that $v_{3}$ represents three right lines through the point $\left(x_{1} y_{1} z_{1}\right)$ meeting $U$ on the conic $3 b d-2 c^{2}$ and the line $c$. Being drawn from a double point on $u_{3}, u_{4}$, they meet the former curve again in three points only,-viz., those common to $u_{3}, \mathrm{U}$, and $c,-\mathrm{a}$ fact verified by the identity

$$
d u_{3} \equiv v_{3}-2 c u_{2}
$$

while they meet $u_{4}$ in six points, other than $x_{1} y_{1} z_{1}$.

$$
\begin{aligned}
& \text { Since } \quad c v_{3} \equiv d^{2}\left(a c-b^{2}\right)+\left(b d-c^{2}\right)\left(b d-2 c^{2}\right) \text {, } \\
& \text { i.e., } \quad \equiv d^{2} u_{4}+u_{2}\left(b d-2 c^{2}\right) \text {, }
\end{aligned}
$$

the six points just mentioned lie on the conic $b d-2 c^{2}$, which has $c$ as its chord of double contact with $b$.

6. In the next place let $U$ represent a proper plane quartic curve; then the equation to the twelve tangents from $\left(x_{1} y_{1} z_{1}\right)$ will be obtained by equating with zero the discriminant of

where

$$
\begin{gathered}
\left(a, b, c, d, e \gamma(\lambda, \mu)^{4},\right. \\
a=\mathrm{U}, \\
4 b=\mathrm{D}_{1} \mathrm{U}, \\
6 c=\frac{1}{2} \mathrm{D}_{1}^{3} \mathrm{U}, \\
4 d=\frac{1}{6} \mathrm{D}_{1}^{3} \mathrm{U}, \\
e=\mathrm{U}_{1} ;
\end{gathered}
$$

$\mathrm{D}_{1}$, as before, standing for the operator $x_{1} \frac{d}{d x}+y_{1} \frac{d}{d y}+z_{1} \frac{d}{d z}$, and $\mathrm{U}_{1}$ for the result of substituting $x_{1}$ for $x ; y_{1}$ for $y$, and $z_{1}$ for $z$, in $U$. Of course $b$ will be a cubic, $c$ a quadratic, and $d$ a linear function of $x y z$, when $a$ or $\mathrm{U}$ is a quartic.

If the standard form of the discriminant of $(a, b, c, d, e \gamma \lambda, \mu)^{4}$, viz. $\mathrm{I}^{8}-27 \mathrm{~J}^{2}$,

where

$$
\begin{aligned}
& \mathrm{I}=a e-4 b d+3 c^{2}, \\
& \mathrm{~J}=a c e+2 b c d-a d^{3}-b^{2} e-c^{3},
\end{aligned}
$$

be transformed into IK $-3 J^{\prime 2}$, by writing

$$
\begin{aligned}
& \mathrm{J}^{\prime}=3 \mathrm{~J}+c \mathrm{I}=4 a c e+2 b c d-3 a d^{2}-3 b^{2} e, \\
& \mathrm{~K}=\mathrm{I}_{t}^{2}+3 c(6 \mathrm{~J}+c \mathrm{I}),
\end{aligned}
$$

then the equation to the twelve tangents from $\left(x_{1} y_{1} z_{1}\right)$ to $U$ may be exhibited under either of the forms

$$
\begin{aligned}
& u_{4} u_{8}^{2}-3 u_{8}^{2}=0 \\
& u_{4}^{3}-27 v_{6}^{2}=0 \\
& \begin{array}{ll}
\text { or } & u_{4}-27 v_{6}^{2}=0 \\
\text { where } & u_{4}=a e-4 b d+3 c^{2}
\end{array} \\
& v_{6}=d c e+2 b c d-a d^{2}-b^{2} e-c^{2} \\
& u_{6}=3 v_{6}+c u_{6} \\
& u_{8}^{\prime}=u_{4}^{2}+3 c\left(6 v_{6}+c u_{4}\right)
\end{aligned}
$$

the suffixes indicating the order of the functions in $x, y, z$. 
7. If $a_{1}, b_{1}, c_{1}, d_{1}$ indicate the results of the substitution of $x_{1}$ for $x$, .$y_{1}$ for $y$, and $z_{1}$ for $z$, in $a \ldots d$ respectively ; then, since

$\begin{array}{lll} & a=\mathrm{U}, & a_{1}=\mathrm{U}_{1}=e ; \\ \text { since } & 4 b=\mathrm{D}_{1} \mathrm{U}, & b_{1}=\mathrm{U}_{1}=e ; \\ \text { since } & 6 c=\frac{1}{3} \mathrm{D}_{1}^{2} \mathrm{U}, & c_{1}=\mathrm{U}_{1}=e ; \\ \text { and since } & 4 d_{1}=\frac{1}{6} \mathrm{D}_{1}^{3} \mathrm{U}, & d_{1}=\mathrm{U}_{1}=e .\end{array}$

It is easily verified, therefore, that $u_{6}, v_{6}$ each pass through $\left(x_{1} y_{1} z_{1}\right)$.

$$
\begin{array}{ll}
\text { Again, } & \mathrm{D}_{1} a=\mathrm{D}_{1} \mathrm{U}=4 b \ldots \ldots \ldots \\
& \mathrm{D}_{1} b=\frac{1}{4} \mathrm{D}_{1}^{2} \mathrm{U}=3 c \ldots \ldots \ldots \\
& \mathrm{D}_{1} c=\frac{1}{12} \mathrm{D}_{1}^{3} \mathrm{U}=2 d \ldots \ldots \\
\mathrm{D}_{1} d=\frac{1}{24} \mathrm{D}_{1}^{4} \mathrm{U}=\mathrm{U}_{1}=e
\end{array}
$$

From these relations it is readily verified that

$$
\begin{aligned}
& \mathrm{D}_{1} u_{4} \equiv 0 \ldots . \\
& \mathrm{D}_{1} v_{6} \equiv 0 .
\end{aligned}
$$

consequently $u_{b}, v_{b}$ represent systems of four and six right lincs respectively drawn through $\left(x_{1} y_{1} z_{1}\right)$.

From (12), (19), (16), it follows at once that

$$
\begin{array}{ll}
\text { and from (17), (18), } & \mathrm{D}_{1}^{2} u_{6}=2 e u_{4}, \\
& \mathrm{D}_{1}^{3} u_{6} \equiv 0 ;
\end{array}
$$

consequently $\left(x_{1} y_{1} z_{1}\right)$ is a quadruple point on $u_{6}$, and $u_{4}$ is the system of four tangents to $u_{6}$ at this point. Further the form of $u_{\theta}$, viz. $3 v_{6}+c u_{6}$, shows that the four lines $u_{4}$ meet $u_{6}$ unly on points common to $u_{6}$ and $v_{6}$, i.e., only at $\left(x_{1} y_{2} z_{1}\right)$, which is therefore a point of inflexion on each of the four branches of $u_{6}$ meeting at that point.

Also, (13), (16), (18), (19),

$$
\begin{aligned}
D_{2} u_{8} & =6 d\left(6 v_{6}+c u_{4}\right)+b c d u_{4} \\
& =12 d\left(3 v_{6}+c u_{6}\right)=12 d u_{6}
\end{aligned}
$$

consequently $u_{8}$ has its first polar with respect to $\left(x_{1} y_{1} z_{1}\right)$, through which it passes, the sextic $u_{6}$ and the line $d$. The point $\left(x_{1} y_{1} z_{1}\right)$ is therefore $a$ quadruple inflexion point on $u_{8}$ also, and the four lines $u_{4}$ are the tangents to $u_{8}$ at that point, each touching one of its four branches in three consecutive points. The form of $u_{8}$, viz.

$$
u_{8}=u_{6}^{2}+3 c\left(6 v_{6}+c u_{6}\right)
$$

shows that the eight points, other than $\left(x_{1} y_{1} z_{1}\right)$, in which its four tangents $u_{4}$ mect it, lie on the conic $c$, which has octuple contact with $u_{3}$ at these points.

8. The curres $u_{6}, u_{8}$ having $\left(x_{1} y_{1} z_{1}\right)$ as a common quadruple-inflexion point, and common tangents at that point, will meet again in only twenty-four points, and the equation (8),

$$
u_{\triangleleft} u_{0}-3 u_{0}^{2}=0
$$


one of the forms of the equation of the twelve tangents drawn from $\left(x_{1} y_{1} z_{1}\right)$ to $\mathrm{U}$, shows that at those points the same twelue lines are double tangents to $u_{8}$.

It may be further shown that these twelve double tangents to $u_{8}$ touch this curve each once at its point of contact with $U$; or, in other words, that $u_{8}$ touches $U$ at the twelve points of contact with the latter curve of their common tangents, the other eight points of intersection of $u_{\mathrm{B}}$ and $\mathrm{U}$ lying on a conic.

For all values of the variables which satisfy $\mathrm{U}=0$, i.e. $a=0$, see (10), (11),

$$
\begin{aligned}
& u_{4}=3 c^{2}-4 b d, \\
& v_{6}=2 b c d-b^{2} e-c^{3} ;
\end{aligned}
$$

whence, and from (12), $u_{6}=(2 c d-b e) b$;

consequently the intersections of $U$, or $a$, and its first polar $b$ lie on $u_{6}$. Again, generally, (13), $u_{8}=u_{4}\left(u_{4}+3 c^{2}\right)+18 c v_{0}$;

so that for all values of $x, y, z$, which satisfy $U$ or $a=0$, substituting the values of $u_{4}, v_{6}$ just above,

$$
\begin{aligned}
u_{8} & =2\left\{\left(3 c^{2}-4 b d\right)\left(3 c^{2}-2 b d\right)+18 b c^{2} d-9 b^{2} c e-9 c^{4}\right\} \\
& =2\left(8 d^{2}-9 c e\right) b^{2} ;
\end{aligned}
$$

consequently, at the twelve points in which $\dot{U}$ meets its first polar $b$, it touches $u_{8}$; the remaining eight points of intersection of $U$ with $u_{8}$ lying on the conic $8 d^{2}-9 c e$.

9. The four tangents $u_{4}$ to $u_{8}$ at the quadruple-infexion point $\left(x_{1} y_{1} z_{1}\right)$ count as twenty-four of the fifty-six tangents which can, in general, be drawn from a point to $u_{8}$. Of the other thirty-two, twenty-four, viz. the twelve double tangents, have been accounted for. In order to find the remaining eight, I have thought it worth while to investigate independently the equation to the thirty-six tangents which can be drawn from $\left(x_{1} y_{1} z_{1}\right)$ considered as a quadruple point only, as may be done without difficulty. It is necessary to form the discriminant of the quartic

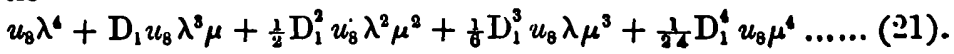

$$
\begin{aligned}
& \text { Now . . } u_{8}=u_{4}^{2}+3 c\left(6 v_{6}+c u_{4}\right) \text {, } \\
& \text { and, (20), } \quad \mathrm{D}_{1} u_{8}=12 d\left(3 v_{6}+c u_{4}\right) \text {; } \\
& \text { whence, (16)-(19), } \frac{1}{2} \mathrm{D}_{1}^{2} u_{8}=6\left\{e\left(3 v_{6}+c u_{4}\right)+2 d^{3} u_{4}\right\} \text {, } \\
& \frac{1}{4} D_{1}^{3} u_{8}=12 d e u_{4} \text {, } \\
& \frac{1}{24} D_{1}^{4} u_{8}=3 e^{2} u_{4} \text {. }
\end{aligned}
$$

Comparing the quartic (21) in $\lambda, \mu$ with (A, B, C, D, EX $\lambda, \mu)$,

$$
\begin{aligned}
& \mathrm{A}=u^{2}+3 c(6 v+c u), \\
& \mathrm{B}=3 d(3 v+c u), \\
& \mathrm{C}=c(3 v+c u)+2 d u, \\
& \mathrm{D}=3 d e u, \\
& \mathrm{E}=3 e^{2} u,
\end{aligned}
$$


the suffixes to $v_{6}$ and $u_{4}$ being dropped for shortness. Hence

$$
\begin{aligned}
& \mathrm{AE}-4 \mathrm{BD}+3 \mathrm{C}^{2}=3\left\{e^{2}\left(u^{3}-27 v^{2}\right)+4\left(c e u-d^{2} u+3 e v\right)^{2}\right\}, \\
& \text { and } \quad \mathrm{ACE}+2 \mathrm{BCD}-\mathrm{AD}^{2}-\mathrm{B}^{2} \mathrm{E}-\mathrm{C}^{3}= \\
& \left\{3 e^{2}\left(u^{3}-27 v^{2}\right)+8\left(c e u-d^{2} u+3 e v\right)^{2}\right\}\left(c e u-d^{3} u+3 e v\right) .
\end{aligned}
$$

10. If for shortness we write

$$
\begin{gathered}
l=e^{2}\left(u_{4}^{8}-27 v_{6}^{2}\right), \\
m=\left(c e-d^{2}\right) u_{4}+3 e v_{6},
\end{gathered}
$$

the equation to the thirty-six tangents from $\left(x_{1} y_{1} z_{1}\right)$ to $u_{8}$ will therefore be

$$
\left(l+4 m^{2}\right)^{3}-\left(3 l+8 m^{2}\right)^{2} m^{2}=0,
$$

which reduces identically to $l^{2}\left(l+3 m^{2}\right)=0$.

Restoring their values for $l, m$, this equation becomes identically

$u_{4}\left(u_{4}^{8}-27 v_{6}^{2}\right)^{2}\left[e^{2}\left\{u_{4}^{2}+3 c\left(6 v_{6}+c u_{4}\right)\right\}-3 d^{2}\left\{2 e\left(3 v_{6}+c u_{4}\right)-d^{2} u_{4}\right\}\right]=0$; i.e., (13), (12), $u_{4}\left(u_{4}^{3}-27 v_{6}^{2}\right)^{2}\left\{e^{2} u_{8}-3 d^{2}\left(2 e u_{0}-d^{2} u_{4}\right)\right\}=0 \quad \ldots .$. (22).

The first factor enters in consequence of the four lines $u_{4}$ meeting the curve each in two points consecutive, on each branch, to the quadruple point. The second factor is the representative of the twelve double tangents; viz., the tangents to U from $\left(x_{1} y_{1} z_{1}\right)$ : lastly, the third factor represents the remaining eight tangents, touching $\imath_{8}$ at the eight points in which it is met by the line $d$, which appeared before as a factor in its first polar (20). These last eight tangents being drawn from a quadruple point will intersect $u_{8}$ again each in two points only lying on the sextic $\quad 2 e u_{6}-d^{2} u_{4}=w_{6}=0$.

This sextic $w_{0}$ has-as appears from its form-sextuple contact with $u_{6}$ at the six points in which they are both met by the line $d$. In fact $w_{6}$ has $\left(x_{1} y_{1} z_{1}\right)$ also as a quadruple-inflexion point, $u_{4}$ being also the four tangents to it at that point. Consequently $w_{6}$ meets $u_{8}$ in twenty-four points other than $\left(x_{1} y_{1} z_{1}\right)$; viz., in the eight common to $u_{8}, u_{4}$ and the conic $c$, besides the sixteen above alluded to. This appears from the identity

$$
e u_{8}-3 c w_{8} \equiv u_{4}\left\{e u_{4}-3 c\left(c e-d^{2}\right)\right\} \text {. }
$$

11. It appears from (13) that the six lines $v_{6}$ meet $u_{8}$ in twenty-four points, other than $\left(x_{1} y_{1} z_{1}\right)$, lying on the quartic

$$
u_{4}+3 c^{2}=0 \text {, }
$$

the other eight points common to this quartic, and $u_{\beta}$ being the eight common to the four lines $u_{4}$ and the conic $c$, which in fact are the eight points of contact with the quartic of its four double tangents $u_{4}$.

12. When the point $\left(x_{1} y_{1} z_{1}\right)$ falls on the curve $U, U_{1}$ or $e=0$, and

$$
\begin{aligned}
& u_{4}=-4 b d+3 c^{2}, \\
& v_{6}=2 b c d-a d^{2}-c^{3}, \\
& u_{6}=(2 b c-3 a d) d, \\
& u_{6}=2\left(8 b^{2}-9 a c\right) d^{2} .
\end{aligned}
$$


In this case, then, $u_{8}$ breaks up into the square of the line d-the tangent to $U$ at the point $\left(x_{1} y_{1} z_{1}\right)$-and the sextic

$$
v_{0}=8 b^{2}-9 a c \text {, }
$$

while, correspondingly, $u_{\theta}$ breaks up into the line $d$ and the quintic

$$
v_{5}=2 b c-3 a d \text {, }
$$

which may be verified to be the first polar of $v_{\theta}$. For since

$$
\begin{aligned}
\mathrm{D}_{1} a & =4 b, \quad \mathrm{D}_{1} b=3 c, \quad \mathrm{D}_{1} c=2 d, \quad \mathrm{D}_{1} d=e, \\
\mathrm{D}_{1} v_{6} & =16 b \mathrm{D}_{1} b-9 a \mathrm{D}_{1} c-9 c \mathrm{D}_{1} a \\
& =48 b c-18 a b-36 b c=6(2 b c-3 a d)=6 v_{5} . \\
\text { Again, } \quad \mathrm{D}_{1} v_{5} & =2 b \mathrm{D}_{1} c+2 c \mathrm{D}_{1} b-3 a \mathrm{D}_{1} d-3 d \mathrm{D}_{1} a \\
& =4 b d+b c^{2}-12 b d=2\left(3 c^{2}-4 b d\right)=2 \iota_{4} ;
\end{aligned}
$$

so that $v_{6}, v_{6}$ have $\left(x_{1} y_{1} z_{1}\right)$ as a common quadruple point and four common tangents at that point; consequently they meet again in ten points only. The ten tangents which can be drawn from $\left(x_{1} y_{1} z_{1}\right)$ to $U$ or $a$ touch $v_{\theta}$ at the same points, as is evident from the form of $v_{6}$; and being drawn from a quadruple point do not meet that curve again.

Prof. Cayley made a few remarks on Mr. Walker's paper, in the course of which he suggested the alteration subsequently adopted by the Anthor.

Mr. S. Roberts then read a paper

\section{On the Order and Singularities of the Parallel of an Algebraical Curve.}

1. A Parallel of a curve is variously defined as the envelope of a circle of constant radius which touches the given curve, or has its centre thereon; and, as the locus of the centre of a circle of constant radius which touches the given curve.

The most obvious property of a Parallel is, that among the normal distances of any point on it from the primitive curve, there is always one of given length, which.I call the Modulus of the Parallel. Moreover, if at any point on the primitive we draw a normal, two points will be determined on the parallel by taking upon the normal lengths equal to the Modulus in opposite directions from the point; and the normals to the Parallel at these points coincide.with the corresponding normal of the Primitive.

The parallel, therefore, has the same normals as the primitive, but each is normal at two points. The latter curve is a parallel to itself to the modulus 0 , but we shall have reason to remark that as the normal distances which determine points on the parallel are measured in two directions, on opposite sides of the corresponding branch of the primitive, this, as parallel to itself, must be reckoned twice.

Since the primitive is included in the family of parallels, they may 